ORTGLAT COMEUTICATIONS.

\section{ON RUPTURE OF THE INNER CIRCLE OF THE IRIS.}

By W. WHITE COOPER, F.R.C.S., Ophthalmic Surgeon to St. Mary's Hospital, etc.

Derachment of the iris from its ciliary border is a common result of external violence; but there is an injury occasionally caused by heary blows upon the eye, which, with its consequences, are but slightly mentioned by the majority of ophthalmic writers. In many systematic works it is passed over altogether. I allude to rupture of the inner margin of the iris, giving rise to wide and persistent dilatation of the pupil and serious disturbance of vision.

The following cases afford illustrations of this severe accident :-

Cass r. In October 1854, a man was admitted into St. Mary's Hospital, having received a very severe blow upon the right eye; the anterior chamber was full of blood, and no trace of the iris was visible. He was put to bed, and cold applications were ordered to be constantly applied to the injured eye; a brisk aperient was administered, and he was placed on low regimen.

I did not see him until the following day, by which time absorption of the effused blood had proceeded so vigorously, that the condition of the iris could be ascertained. It was then seen that this blood had issued from two fissures in the pupillary margin of the iris, which presented a jagged appearance. The pupil was widely dilated, perfectly motionless, and the sight was so far impaired that large objects only could be discerned.

The eye was, however, free from inflammation, and the patient made no complaint of pain. Under these circumstances, as there happened to be a great demand for beds, the man was permitted to leave the hospital, under the promise of attending as an out-patient. This, however, he did not think proper to do; and it was only by accident that I subsequently obtained an opportunity of examining the eye. Its condition at the expiration of two months was as follows :-

The pupil was still widely dilated, and the irregularity of the inner margin of the iris was very distinctly seen.

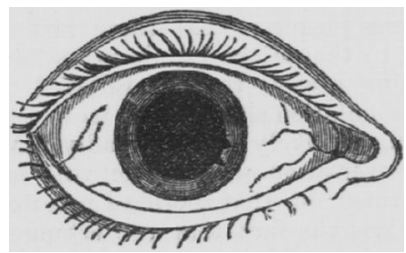

Fig. 1.

Neither contraction nor dilatation of the iris could be excited; it remained perfectly motionless under every amount of light. The eye had speedily recorered from the accident, with the exception of the sight, which remained very dull, all objects appearing hazy and indistinct. The sight was improved by looking through a pin-hole aperture in a card. As there were no indications of inflammatory action, he was recommended to supply himself with a pierced diaphragm to limit the quantity of light entering the eye, and to aroid everything which could over-excite the organ.

CASE Ir. An officer of Dragoons, quartered in Dublin, early in October 1854, was playing at racquets, when he received a violent blow from the ball on the left eye. For 2 time he was stunned, but on regaining his senses ho found that sight had left the injured eje. He placed himself under the care of an eminent physician, and Mr. Wildo
Wes also consulted, so that no gkill we wenting in the early treatment of the case.

This gentleman was seen by mo on the 16th of the following November, about six weeks after the accident. The condition of the eje was as follows:-The pupil was 80 condition of the eye was the iris was reduced to a mere narrow strip, and was perfectly immoreable under the strongest light. The lower portion of the margin presented two fissures, the edges of which being drawn asunder gare a saw-like appearance to that part. The sight was very

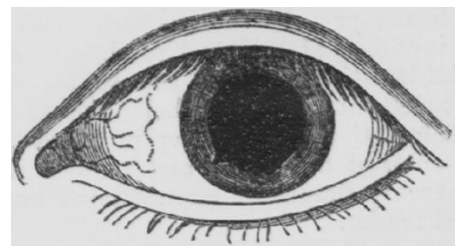

Fig. 2.

imperfect, large objects only being discernible ; it was not improved by a pierced diaphragm.

Stimulating embrocations and instillation of tinctures of aconite and of opium were tried, but without the slightest benefit.

I saw this gentleman from time to time, and six weeks after his first visit decided improvement in the sight displayed itself : by looking through a pin-hole aperture ho was able to discern objects which had before been very indistinct. After the lapse of four months, an alteration became visible in the condition of the iris; a certain power of contraction had been regained, for there was a marked diminution in the size of the pupil, and an equally marked increase in the breadth of the iris. Still it could not be seen to act under the influence of light, there being no discernible alteration when the eye was alternately shaded and exposed.

In July, when I last saw the patient, a still further improvement had taken place, and now a faint contraction and dilatation could be excited. The sight was materially amended, for not only could he discern distant objects by means of the pierced diaphragm, but he could read and write with tolerable facility. There was, however, this drawback, that under the most favourable circumstances the vision of the two eyes was not equal; and as my patient could not constantly wear the diaphragm, he unconsciously acquired the habit of closing the injured eye when looking at any object.

Case III. On the 14th of June 1855, I was consulted respecting the case of a youth, aged 17 years, who had received the following injury eleven weeks previously : a round and heavy pebble was thrown by a lad at another boy who had incensed him, but the missile unfortunately lighted full on the right eye of my patient, who happened accidentally to come between the two at that unlucky moment. He fell from the force of the blow, and was at once rendered blind of that eye. He was placed under medical care, and active measures were resorted to; but the sight was only restored to a very imperfect extent. He was therefore brought to town.

The nature of the case was unmistakeable. The pupil presented the characteristic wide dilatation, and the lower margin had that serrated appearance which has already been described. In this case there were three fissures, so that the irregularities were very marked. The colour of the iris was unchanged, and the eye was perfectly free from inflammation. On being desired to close the sound eye, it was ascertained that he could see large objects, but even these indistinctly. On placing a pin-hole aperture before the eye, he could recognise features, and read tolerably large type.

As the injury was extensive, I formed an unfavourable opinion as to the result of this case, and advised that$$
\xi
$$ 
incteid of his being sppreaticed to $\&$ printer (es mas incended), come agricultural occupation, or other pursuit roguiring little exertion of the sight, should be selected. the use of a piarced diaphragm was recommended.

Bexares. It is well known that clean incisions of the iris are seldom followed by inflammation, and an eye in which the iris has been ruptured by injury may be fortunate enough to escape : but when we consider the great violence done to the whole organ, the possibility of deepsested effusion, and of subsequent insidious inflammation, such cases require to be closely watched. The effused blood which always veils the iris will speedily disappear under the influence of cold and of simple treatment, but strict antiphlogistic measures are to be enforced from the commencement; and if there should arise indications of inflammation, as pain deep in the eye, or about the brow, tenderness of the globe, and zonular redness of the sclerotic, mercury should be administered until the gums are rendered tender.

In the cases under consideration, the dilatation of the pupil is as great as that produced by a strong solution of atropine (which is always attended with considerable confusion of sight) ; but the impairment of vision is for a long time after the accident far greater than can be accounted for by any such dilatation. This is not surprising, when the character of the injury is considered. A blow suffciently violent to cause rupture of the iris, is likely to produce concussion of the retina, and separation of that membrane might even take place; again, chronic inflammation of the retina may be excited. If concussion merely, the effects will gradually pass away, and the improvement of vision through a pin-hole aperture will be the index by which amendment can be traced. If inflammation has been excited, the injury to vision may be permanent.

So far as my experience has hitherto gone, injuries of the pupillary margin of the iris are little amenable to treatment. This will, no doubt, in a great degree depend upon the depth to which the fissure extends, and the consequent amount of laceration of the fibres which contract the pupil. If these are completely divided, the pupil will be widely expanded, and no application will cause its contraction; if only partially divided, a certain amount of contraction may be excited. The question will be anxiously asked, Is the eye likely to recorer? for although after a time the organ becomes accustomed to the innatural glare of light, the sight is under the most favourable circumstances seriously interfered with. A very cautious prognosis should be given. Time perhaps may improve the state of things; but if the laceration be extensive, it must be very doubtful whether the powers of reparation will be sufficient to bring the eye into a useful condition. The edges of the fissures are widely separated, and cannot be approximated by any means with which I am acquainted. The fissure may be likened to a cleft palate; but we are without the means which modern skill has supplied, of removing that defect by operation.

But do we possess any means of remedying the inconvenience arising from this permanent mydriasis? Unquestionably! by artificially imitating the contracted pupil, the eye may be rendered useful, unless damaged by inflammation. This is best done by means of a spectacle-frame, fitted for the affected eye with an opaque plate, either of thin steel, horn, or blackened tortoiseshell, and having in the centre, to correspond with the pupil, an aperture, either circular, or as a transverse slit. The form and exact dimensions must be a matter of experiment. Various forms and sizes should be tried, and that selected which affords the best vision.*

Iondon, October 1855.

- Messrs. Carpenter and Westley, of 24, Regent Stroet, hare had much experience in such cases.

\section{1}

\section{ON BRIGHT'S DISSEASE.}

By ROBERT C. R. JORDAN, M.B., etc.

[Read before the Medical Society of Queen's College,

Tas intention of this paper is to bring before this Societs a brief account of the symptoms, pathology, and treatment of those disenses of the kidney known under the common name of Bright's disease. In doing this, if the opinion here given differ slightly from those of authors on the subject, my hearers must remember, in judging me, that no two of these authors agree amongst themselves.

It is strange that whilst the minute anatomy of no other gland is so thoroughly understood as that of the kidney, and whilst, moreover, it has this additional adrantage that its secretion can be collected and examined, there is yet more discrepancy in the various accounts of its diseases than in those of any other organ.

I said that its secretion can be collected and examined; no one doubts the utility of this as a means of diagnosis. The microscope and the test tube are to the diseases of the kidney what the stethoscope is to disease of the lung.

Bright's disease was not recognised before the early days of tho physician from whom it takes its name; but the first step towards the discovery was made by Dr. Blackall, who was the first to detect the presence of albumen in the urine. Dr. Bright first saw the importance of this with regard to kidney disease, as also the importance of renal disease in producing dropsy, and, indeod, other morbid phenomens. His first case was taken in the year 1825, exactly thirty years ago. Since this time, many able men have worked at the history of these diseases, but the subject is yet far from exhausted, nor indeed has the attontion given to it been as general as it ought; eren in one of Dr. Graves' clinical lectures published as recently as 1848, he labours strenuously to prove that the albuminuria in Bright's disease is not a sequence of the changes in the kidney.

But though no pathologist who has ever studied the appearances of the kidney and the state of disease which had preceded them could now doubt this, yet there is an error, which must be avoided, of the opposite character. Albuminuria and Bright's disease are not synonyms, and albumen in the urine is far from being a pathognomonic symptom of the disease in question. Albumen may be produced by pus, from either the kidney, ureter, bladder, urethra or vagina; by blood, from either of the samo localities, even, as stated by Christison, by particular kinds of food, in some people. That these last cases are very rare, is proved by that of the Edinburgh student, quoted by Christison, being always the one referred to. This rare effect of malassimilation should lead us to look on the case with suspicion, as likely to end badly. My friend Dr. Wade, in a conversation with me on this subject a short time ago, informed me that Bernard was wont to demonstrate to his class the fact that the albumen of eggs, injected into the reins of dogs, was got rid of by the kidneys, in the form of albumen, coagulable by heat.

Spermatorrhoea has again been given as a cause of albuminuria. Though a modified form of albumen, or a compound allied to it, exists in the seminal secretion, yet it is not coagulated by heat. But it is possible that the diseased secretion may be slightly modified, and that this may then be the case. Albumen may also occur in the urine from congestion, and yet the kidney not be primarily in fault. Thus, in cardiac affections, albuminuria maJ arise from the mechanical congestion of the organ. It requires, therefore, other evidence besides the presence of albumen in the urine to decide on the existence of renal disease.

Near the close of the disease, the albumen often gets much less in quantity. The cause of this is easily found in the appearance of the kidney at that stage of the illness. The albumen is rather, by its quantity, a measure of the The albumen is rather, by of the progress of the disease. Indeed, the albumen rather lessens than increases as the 